\title{
EVALUACIÓN DE LA FIABILIDAD Y VALIDEZ DEL CUESTIONARIO DE SALUD MENTAL POSITIVA EN PROFESORES UNIVERSITARIOS DE ENFERMERÍA EN CATALUÑA
}

\section{BÁRBARA HURTADO-PARDOS ${ }^{1}$, TERESA LLUCH-CANUT², IRMA CASAS GARCÍA ${ }^{3}$, CARLOS SEQUEIRA ${ }^{4}$, MONTSE PUIG-LLOBET ${ }^{5}$ Y JUAN ROLDÁN-MERINO 6}

${ }_{1}^{1}$ Profesora titular. Campus Docente Sant Joan de Déu-Fundación Privada. Adscrita a la Universitat de Barcelona. ${ }^{2}$ Catedrática de Enfermería Psicosocial y Salud Mental. Escuela de Enfermería de la Universitat de Barcelona. ${ }^{3}$ Profesora. Universitat Autònoma de Barcelona. Hospital Universitari Germans Trias i Pujol. Badalona (Barcelona).

${ }^{4}$ Profesor coordinador. Escola Superior de Enfermagem do Porto. Oporto, Portugal.

${ }^{5}$ Directora del Departamento de Enfermería de Salud Pública, Salud Mental y Maternoinfantil. Escuela de Enfermería de la Universitat de Barcelona.

${ }^{6}$ Profesor titular. Campus Docente Sant Joan de Déu-Fundación Privada. Adscrita a la Universitat de Barcelona.

\section{RESUMEN}

Introducción: el objetivo de este estudio fue analizar la fiabilidad y validez del Cuestionario de Salud Mental Positiva (CSMP) en una muestra de profesores universitarios de Enfermería en Cataluña.

Método: se realizó un estudio transversal en 263 docentes universitarios de las diferentes escuelas o facultades de Enfermería de Cataluña. Se analizó la fiabilidad del CSMP mediante el coeficiente alfa de Cronbach. Para el análisis de la validez convergente y discriminante, se obtuvieron los coeficientes de correlación entre factores y con la puntuación total del cuestionario. La validez discriminante también se analizó mediante el cuestionario General Health Questionnaire (GHQ-12), que evalúa el malestar psicológico experimentado durante los 30 días previos al momento de la evaluación.

Resultados: el CSMP ha demostrado una buena consistencia interna, y la validez convergente y discriminante han sido satisfactorias.

Conclusiones: este cuestionario es válido y fiable para valorar la salud mental positiva en docentes universitarios de Enfermería.

Palabras clave: salud mental positiva, fiabilidad y validez, instrumento. 


\section{INTRODUCCIÓN}

En el año 2001, la Organización Mundial de la Salud (OMS) definió la salud mental como «un estado de bienestar en el cual el individuo es consciente de sus propias capacidades, puede afrontar las tensiones normales de la vida, puede trabajar de forma productiva y fructífera y es capaz de hacer una contribución a su comunidad» ${ }^{1}$. Con esta definición, se contribuyó a reforzar la definición de salud de principios del siglo XX, no siendo solo la ausencia de enfermedad. Desde la psicología, ha surgido con fuerza la parte positiva de la salud mental, destacando la necesidad de potenciar y reforzar las fortalezas del ser humano ${ }^{2,3}$. Con la finalidad de poder desarrollar intervenciones dirigidas a la promoción de la salud mental positiva (SMP), es necesario disponer de modelos conceptuales que orienten las acciones que seguir, así como de instrumentos de valoración que permitan medir los niveles de SMP antes y después de la intervención. En el año 1999, Lluch realizó una investigación centrada en el estudio del constructo SMP formulado por Jahoda ${ }^{5}$ (1958), con dos objetivos complementarios: definir un modelo conceptual de SMP y construir un instrumento, el Cuestionario de Salud Mental Positiva (CSMP), tanto para operativizar el modelo conceptual como también para poder evaluar la SMP. El resultado de dicha investigación fue la formulación de un modelo multifactorial de SMP configurado por seis factores, que, sumados, definen el constructo SMP: satisfacción personal (F1), actitud prosocial (F2), autocontrol (F3), autonomía (F4), resolución de problemas y autoactualización (F5) y habilidades de relación interpersonal (F6) ${ }^{4,6}$ (tabla 1).

EI CSMP está siendo utilizado por diversos equipos de investigación, especialmente, en España, Latinoamérica y Portugal7,11. Sin embargo, las propiedades psicométricas se han analizando en muy pocos estudios $8-11$.

Correspondencia: Teresa Lluch-Canut

Correo electrónico: tlluch@ub.edu
La necesidad de hacer crecer la robustez métrica de este cuestionario viene justificada no solo por su uso, sino también por la escasez de cuestionarios específicos que evalúen el constructo de SMP12.

Por todo esto, el objetivo de este estudio fue analizar la fiabilidad y validez del CSMP en una muestra de profesores universitarios de Enfermería en Cataluña.

\section{MÉTODO}

\section{Diseño}

Estudio transversal de fiabilidad y validez del CSMP.

\section{Población y muestra}

Las propiedades métricas se analizaron en una muestra voluntaria de 263 profesores universitarios de Enfermería de las escuelas/universidades de Enfermería en Cataluña (España).

Los criterios de inclusión fueron profesores de Enfermería con un mínimo de un año de experiencia en el ámbito docente y que se encontraran en activo en el momento del estudio.

\section{Variables e instrumentos de medida}

Se utilizó un formulario demográfico para recoger datos sociodemográficos de los participantes. Las variables que se recogieron fueron: edad, sexo, categoría docente y años en la universidad.

El CSMP de Lluch consta de 39 ítems agrupados en seis factores que definen el constructo: satisfacción personal; actitud prosocial; autocontrol; autonomía; resolución de problemas y actualización, y habilidades interpersonales de relación. Cada ítem se responde mediante una escala que va de 1 a 4 , de acuerdo con la frecuencia con que se producen: siempre o casi siempre, muy a menudo, a veces, nunca o casi nunca. A mayor puntuación para el total del CSMP y de cada uno de los factores, mayor 
Tabla 1. Distribución por factores de los ítems del Cuestionario de Salud Mental Positiva (CSMP) y valores máximos y mínimos de cada factor y del cuestionario global

\begin{tabular}{|c|c|c|}
\hline Factores & Ítems & Valores máximos y mínimos \\
\hline F1: satisfacción personal & $4^{*}, 6,7,12,14,31,38,39$ & 8-32 \\
\hline F2: actitud prosocial & $1,3,23^{*}, 25^{*}, 37^{*}$ & $5-20$ \\
\hline F3: autocontrol & $2,5^{*}, 21^{*}, 22^{*}, 26^{*}$ & $5-20$ \\
\hline F4: autonomía & $10,13,19,33,34$ & $5-20$ \\
\hline $\begin{array}{l}\text { F5: resolución de problemas y } \\
\text { autoactualización }\end{array}$ & $\begin{array}{c}15^{*}, 16^{*}, 17^{*}, 27^{*}, 28^{*}, 29^{*}, \\
32^{*}, 35^{\star}, 36^{*}\end{array}$ & $9-36$ \\
\hline $\begin{array}{l}\text { F6: habilidades de relación } \\
\text { interpersonal }\end{array}$ & $8,9,11^{*}, 18^{*}, 20 *, 24,30$ & $7-28$ \\
\hline CSMP total & Del 1 al 39 & $39-156$ \\
\hline
\end{tabular}

*Ítems invertidos.

nivel de SMP. La distribución de los 39 ítems del CSMP se muestra en la tabla 1. El CSMP fue validado inicialmente en una muestra de estudiantes de Enfermería6.

También se utilizó el cuestionario General Health Questionnaire $^{13}$ (GHQ-12). Este cuestionario evalúa el malestar psicológico experimentado durante los 30 días previos al momento de la evaluación y permite obtener una puntuación total de 12, que se interpreta como indicador de percepción de malestar psicológico cuando esta puntuación es superior a 2. Este cuestionario se utilizó para analizar la validez discriminante.

Los diferentes instrumentos se diseñaron en formato online y fueron administrados durante el período de junio a diciembre de 2013.

\section{Consideraciones éticas}

El estudio fue aprobado por el Comité Ético de Inves tigación Clínica de la Fundació Sant Joan de Déu, con el código asignado CEIC PIC-65-13, y por el comité Ético de Investigación del Campus Docente Sant
Joan de Déu. Los participantes fueron informados sobre la autoría y el propósito de la investigación. Se aseguró que se mantendría el anonimato y la confidencialidad de todos los datos obtenidos.

\section{Análisis estadístico}

En primer lugar, se hizo un análisis estadístico de todas las variables incluidas en el estudio. Para cada una de las variables cualitativas, se calcularon frecuencias y porcentajes y, para cada una de las variables cuantitativas, la media y desviación típica.

La fiabilidad del cuestionario se analizó mediante el coeficiente alfa de Cronbach; sus valores están comprendidos entre 0 y 1 , y se acepta que el valor ha de ser igual o superior a 0,70 para considerar que el cuestionario tiene una buena consistencia interna. Se calculó también el coeficiente de homogeneidad de los ítems corregido ${ }^{14}$, estimando las correlaciones de cada ítem con el total del cuestionario y con cada factor y aceptando como límite inferior una correlación de 0,20. También se analizó la correlación del CSMP mediante el coeficiente de corre- 
lación de Pearson ( $r$ ), entre las diferentes dimensiones del cuestionario y también entre el resultado de cada uno de los factores y el total, evaluando, así, la validez convergente y discriminante, partiendo de la hipótesis de que la correlación entre cada factor y la puntuación total del cuestionario debe ser más fuerte que las correlaciones entre los factores ${ }^{15}$.

La validez discriminante también se analizó mediante la comparación de las medias de las dimensiones del CSMP con el GHQ-12 (inferior o igual a 2 y superior a 2) mediante la prueba de la $t$ de StudentFisher para muestras independientes ${ }^{13}$.

Para el análisis de los datos, se utilizó el programa estadístico SPSS para Windows (IBM SPSS versión 19.0; IBM Corp., Armonk, Nueva York, Estados Unidos).

\section{RESULTADOS}

Todos los cuestionarios fueron cumplimentados por 263 profesores universitarios de Enfermería. La media de edad de los participantes fue de 47,8 años (desviación estándar [DE]: 9,9) y la gran mayoría eran mujeres (79,1\%). El 50,6\% de los docentes que participaron eran profesores titulares de universidad. Un 32,3\% declararon tener menos de cinco años de antigüedad; un 25,5\%, entre seis y 15 años de antigüedad; y un 42,2 \% tenía más de 15 años de antigüedad.

\section{Análisis de los ítems}

El valor medio de cada ítem varió de 1,90 (ítem 34) a 3,54 (ítem 17) y la DE varió de 0,59 (ítem 37) a 1,06 (ítem 3 e ítem 19). El ítem con el valor porcentual de techo más alto fue el ítem 23 («pienso que soy una persona digna de confianza») $(79,8 \%)$ y los ítems con la clasificación del suelo porcentual más alto fueron el ítem 33 («me resulta difícil tener opiniones personales») (62,05\%) y el ítem 3 («a mí, me resulta especialmente difícil escuchar a las personas que me cuentan sus problemas») $(48,7 \%)$ (tabla 2).

Tabla 2. Estadísticos descriptivos de los ítems del Cuestionario de Salud Mental Positiva (media, desviación estándar [DE], porcentaje suelo y porcentaje techo)

\begin{tabular}{l|l|l|l|l|l|}
\hline Contenido de los ítems resumidos & Media & DE & $\%$ suelo & $\%$ techo \\
\hline F1: satisfacción personal & & & & \\
\hline P4 & Me gusto como soy & 3,24 & 0,77 & 0,4 & 43,7 \\
\hline P6 & Me siento a punto de explotar & 2,87 & 0,76 & 0,8 & 22,4 \\
\hline P7 & Para mí, la vida es aburrida y monótona & 2,95 & 0,74 & 1,5 & 23,6 \\
\hline P12 & Veo mi futuro con pesimismo & 2,89 & 0,72 & 0,8 & 20,2 \\
\hline P14 & $\begin{array}{l}\text { Me considero una persona menos importante que el } \\
\text { resto de personas que me rodean }\end{array}$ & 2,90 & 0,76 & 2,7 & 21,7 \\
\hline P31 & Creo que soy un/a inútil y no sirvo para nada & 2,90 & 0,76 & 3,0 & 21,3 \\
\hline P38 & Me siento insatisfecha/o conmigo misma/o & 2,75 & 0,67 & 3,0 & 10,6 \\
\hline P39 & Me siento insatisfecha/o de mi aspecto físico & 2,65 & 0,75 & 3,8 & 12,9 \\
\hline
\end{tabular}


Tabla 2. Estadísticos descriptivos de los ítems del Cuestionario de Salud Mental Positiva (media, desviación estándar [DE], porcentaje suelo y porcentaje techo) (cont.)

\begin{tabular}{|c|c|c|c|c|c|}
\hline \multicolumn{2}{|c|}{ Contenido de los ítems resumidos } & Media & DE & $\%$ suelo & $\%$ techo \\
\hline \multicolumn{6}{|c|}{ F2: actitud prosocial } \\
\hline P1 & $\begin{array}{l}\text { Me resulta especialmente difícil aceptar a los otros } \\
\text { cuando tienen actitudes distintas a las mías }\end{array}$ & 2,22 & 0,85 & 20,5 & 7,2 \\
\hline P3 & $\begin{array}{l}\text { A mí, me resulta especialmente difícil escuchar a las } \\
\text { personas que me cuentan sus problemas }\end{array}$ & 1,91 & 1,06 & 48,7 & 12,9 \\
\hline P23 & Pienso que soy una persona digna de confianza & 3,67 & 0,78 & 6,5 & 79,8 \\
\hline P25 & Pienso en las necesidades de los demás & 3,37 & 0,80 & 3,4 & 54,0 \\
\hline P37 & Me gusta ayudar a los demás & 3,52 & 0,59 & 1,5 & 56,3 \\
\hline \multicolumn{6}{|c|}{ F3: autocontrol } \\
\hline P2 & Los problemas me bloquean fácilmente & 2,26 & 0,98 & 23,6 & 14,8 \\
\hline P5 & $\begin{array}{l}\text { Soy capaz de controlarme cuando experimento } \\
\text { emociones negativas }\end{array}$ & 3,08 & 0,77 & 3,4 & 30,4 \\
\hline P21 & $\begin{array}{l}\text { Soy capaz de controlarme cuando tengo pensamientos } \\
\text { negativos }\end{array}$ & 3,43 & 0,72 & 1,1 & 55,5 \\
\hline P22 & $\begin{array}{l}\text { Soy capaz de mantener un buen nivel de autocontrol } \\
\text { en las situaciones conflictivas de mi vida }\end{array}$ & 3,41 & 0,69 & 0,4 & 52,5 \\
\hline P26 & $\begin{array}{l}\text { Si estoy viviendo presiones exteriores desfavorables, } \\
\text { soy capaz de continuar manteniendo mi equilibrio } \\
\text { personal }\end{array}$ & 3,13 & 0,73 & 1,1 & 33,1 \\
\hline \multicolumn{6}{|c|}{ F4: autonomía } \\
\hline P10 & Me preocupa mucho lo que los demás piensan de mí & 2,27 & 0,90 & 18,3 & 12,5 \\
\hline P13 & $\begin{array}{l}\text { Las opiniones de los demás me influyen mucho a la } \\
\text { hora de tomar mis decisiones }\end{array}$ & 1,97 & 0,91 & 34,2 & 8,7 \\
\hline P19 & Me preocupa que la gente me critique & 2,42 & 1,06 & 22,4 & 22,1 \\
\hline P33 & Me resulta difícil tener opiniones personales & 1,66 & 1,01 & 62,0 & 11,8 \\
\hline P34 & $\begin{array}{l}\text { Cuando tengo que tomar decisiones importantes, me } \\
\text { siento muy insegura/o }\end{array}$ & 1,90 & 0,90 & 37,6 & 8,7 \\
\hline
\end{tabular}


Tabla 2. Estadísticos descriptivos de los ítems del Cuestionario de Salud Mental Positiva (media, desviación estándar [DE], porcentaje suelo y porcentaje techo) (cont.)

\begin{tabular}{|c|c|c|c|c|c|}
\hline \multicolumn{2}{|c|}{ Contenido de los ítems resumidos } & Media & $\mathrm{DE}$ & $\%$ suelo & $\%$ techo \\
\hline \multicolumn{6}{|c|}{ F5: resolución de problemas y actualización } \\
\hline P15 & Soy capaz de tomar decisiones por mí misma/o & 3,62 & 0,68 & 2,7 & 70,3 \\
\hline P16 & $\begin{array}{l}\text { Intento sacar los aspectos positivos de las cosas malas } \\
\text { que me suceden }\end{array}$ & 3,40 & 0,73 & 1,5 & 53,6 \\
\hline P17 & Intento mejorar como persona & 3,54 & 0,63 & 2,3 & 59,7 \\
\hline P27 & Cuando hay cambios en mi entorno, intento adaptarme & 3,42 & 0,63 & 0,8 & 49,0 \\
\hline P28 & $\begin{array}{l}\text { Delante de un problema, soy capaz de solicitar } \\
\text { información }\end{array}$ & 3,25 & 0,69 & 1,1 & 38,4 \\
\hline P29 & $\begin{array}{l}\text { Los cambios que ocurren en mi rutina habitual me } \\
\text { estimulan }\end{array}$ & 2,96 & 0,89 & 7,2 & 30,4 \\
\hline P32 & Trato de desarrollar y potenciar mis buenas aptitudes & 3,41 & 0,64 & 1,5 & 48,3 \\
\hline P35 & Soy capaz de decir no cuando quiero decir no & 2,90 & 0,78 & 4,2 & 22,1 \\
\hline P36 & $\begin{array}{l}\text { Cuando se me plantea un problema, intento buscar } \\
\text { posibles soluciones }\end{array}$ & 3,51 & 0,62 & 1,9 & 55,9 \\
\hline \multicolumn{6}{|c|}{ F6: habilidades de relación interpersonal } \\
\hline P8 & $\begin{array}{l}\text { A mí, me resulta especialmente difícil dar apoyo } \\
\text { emocional }\end{array}$ & 3,00 & 0,80 & 2,3 & 30,0 \\
\hline P9 & $\begin{array}{l}\text { Tengo dificultades para establecer relaciones } \\
\text { interpersonales profundas y satisfactorias con algunas } \\
\text { personas }\end{array}$ & 2,82 & 0,78 & 2,7 & 20,9 \\
\hline P11 & $\begin{array}{l}\text { Creo que tengo mucha capacidad para ponerme en el } \\
\text { lugar de los demás y comprender sus respuestas }\end{array}$ & 3,30 & 0,69 & 0,4 & 43,3 \\
\hline P18 & Me considero «un/a buen/a psicólogo/a» & 2,98 & 0,83 & 1,5 & 31,9 \\
\hline P20 & Creo que soy una persona sociable & 3,53 & 0,62 & 0,0 & 60,1 \\
\hline P24 & $\begin{array}{l}\text { A mí, me resulta especialmente difícil entender los } \\
\text { sentimientos de los demás }\end{array}$ & 2,89 & 0,79 & 3,0 & 23,6 \\
\hline P30 & $\begin{array}{l}\text { Tengo dificultades para relacionarme abiertamente con } \\
\text { mis profesores/jefes }\end{array}$ & 3,00 & 0,74 & 0,4 & 27,8 \\
\hline
\end{tabular}

\section{Fiabilidad}

El coeficiente de consistencia interna alfa de Cronbach para el total de la escala fue de 0,885. En cinco de los seis factores, los valores fueron próximos o supe- riores a 0,700. El factor 2 (actitud prosocial) obtuvo un coeficiente de 0,338. También se calculó el alfa de Cronbach excluyendo cada ítem de la escala y no se observó que la exclusión mejorase la consistencia interna del cuestionario (tabla 3). 
Tabla 3. Coeficiente de consistencia interna (alfa de Cronbach) del Cuestionario de Salud Mental Positiva

\begin{tabular}{|c|c|c|c|c|}
\hline \multirow{2}{*}{\multicolumn{2}{|c|}{ Contenido de los ítems resumidos }} & \multicolumn{3}{|c|}{ Alfa de Cronbach } \\
\hline & & $\begin{array}{l}\text { Total de la } \\
\text { subescala }\end{array}$ & $\begin{array}{l}\text { Total de la } \\
\text { subescala } \\
\text { sin el ítem }\end{array}$ & $\begin{array}{l}\text { Total de la } \\
\text { escala sin } \\
\text { el ítem }\end{array}$ \\
\hline \multicolumn{2}{|c|}{ F1: satisfacción personal } & 0,633 & & \\
\hline P4 & Me gusto como soy & & 0,627 & 0,854 \\
\hline P6 & Me siento a punto de explotar & & 0,609 & 0,854 \\
\hline P7 & Para mí, la vida es aburrida y monótona & & 0,586 & 0,850 \\
\hline P12 & Veo mi futuro con pesimismo & & 0,555 & 0,849 \\
\hline P14 & $\begin{array}{l}\text { Me considero una persona menos importante que el } \\
\text { resto de personas que me rodean }\end{array}$ & & 0,600 & 0,851 \\
\hline P31 & Creo que soy un/a inútil y no sirvo para nada & & 0,629 & 0,855 \\
\hline P38 & Me siento insatisfecha/o conmigo misma/o & & 0,607 & 0,854 \\
\hline P39 & Me siento insatisfecha/o de mi aspecto físico & & 0,589 & 0,852 \\
\hline \multicolumn{2}{|c|}{ F2: actitud prosocial } & 0,338 & & \\
\hline P1 & $\begin{array}{l}\text { Me resulta especialmente difícil aceptar a los otros } \\
\text { cuando tienen actitudes distintas a las mías }\end{array}$ & & 0,234 & 0,848 \\
\hline P3 & $\begin{array}{l}\text { A mí, me resulta especialmente difícil escuchar a las } \\
\text { personas que me cuentan sus problemas }\end{array}$ & & 0,236 & 0,847 \\
\hline P23 & Pienso que soy una persona digna de confianza & & 0,292 & 0,852 \\
\hline P25 & Pienso en las necesidades de los demás & & 0,300 & 0,853 \\
\hline P37 & Me gusta ayudar a los demás & & 0,361 & 0,855 \\
\hline \multicolumn{2}{|c|}{ F3: autocontrol } & 0,623 & & \\
\hline P2 & Los problemas me bloquean fácilmente & & 0,805 & 0,850 \\
\hline P5 & $\begin{array}{l}\text { Soy capaz de controlarme cuando experimento } \\
\text { emociones negativas }\end{array}$ & & 0,598 & 0,855 \\
\hline P21 & $\begin{array}{l}\text { Soy capaz de controlarme cuando tengo pensamientos } \\
\text { negativos }\end{array}$ & & 0,457 & 0,851 \\
\hline P22 & $\begin{array}{l}\text { Soy capaz de mantener un buen nivel de autocontrol en } \\
\text { las situaciones conflictivas de mi vida }\end{array}$ & & 0,427 & 0,849 \\
\hline P26 & $\begin{array}{l}\text { Si estoy viviendo presiones exteriores desfavorables, soy } \\
\text { capaz de continuar manteniendo mi equilibrio personal }\end{array}$ & & 0,465 & 0,853 \\
\hline
\end{tabular}


Tabla 3. Coeficiente de consistencia interna (alfa de Cronbach) del Cuestionario de Salud Mental Positiva (cont.)

\begin{tabular}{|c|c|c|c|c|}
\hline \multirow{2}{*}{\multicolumn{2}{|c|}{ Contenido de los ítems resumidos }} & \multicolumn{3}{|c|}{ Alfa de Cronbach } \\
\hline & & $\begin{array}{l}\text { Total de la } \\
\text { subescala }\end{array}$ & $\begin{array}{l}\text { Total de la } \\
\text { subescala } \\
\text { sin el ítem }\end{array}$ & $\begin{array}{l}\text { Total de la } \\
\text { escala sin } \\
\text { el ítem }\end{array}$ \\
\hline \multicolumn{2}{|c|}{ F4: autonomía } & 0,831 & & \\
\hline P10 & Me preocupa mucho lo que los demás piensan de mí & & 0,799 & 0,850 \\
\hline P13 & $\begin{array}{l}\text { Las opiniones de los demás me influyen mucho a la } \\
\text { hora de tomar mis decisiones }\end{array}$ & & 0,778 & 0,846 \\
\hline P19 & Me preocupa que la gente me critique & & 0,827 & 0,854 \\
\hline P33 & Me resulta difícil tener opiniones personales & & 0,797 & 0,848 \\
\hline P34 & $\begin{array}{l}\text { Cuando tengo que tomar decisiones importantes, me } \\
\text { siento muy insegura/o }\end{array}$ & & 0,786 & 0,850 \\
\hline \multicolumn{2}{|c|}{ F5: resolución de problemas y actualización } & 0,763 & & \\
\hline P15 & Soy capaz de tomar decisiones por mí misma/o & & 0,708 & 0,851 \\
\hline P16 & $\begin{array}{l}\text { Intento sacar los aspectos positivos de las cosas malas } \\
\text { que me suceden }\end{array}$ & & 0,724 & 0,851 \\
\hline P17 & Intento mejorar como persona & & 0,731 & 0,851 \\
\hline P27 & Cuando hay cambios en mi entorno, intento adaptarme & & 0,718 & 0,850 \\
\hline P28 & Delante de un problema, soy capaz de solicitar información & & 0,762 & 0,856 \\
\hline P29 & $\begin{array}{l}\text { Los cambios que ocurren en mi rutina habitual me } \\
\text { estimulan }\end{array}$ & & 0,770 & 0,854 \\
\hline P32 & Trato de desarrollar y potenciar mis buenas aptitudes & & 0,734 & 0,850 \\
\hline P35 & Soy capaz de decir no cuando quiero decir no & & 0,798 & 0,856 \\
\hline P36 & $\begin{array}{l}\text { Cuando se me plantea un problema, intento buscar } \\
\text { posibles soluciones }\end{array}$ & & 0,711 & 0,850 \\
\hline \multicolumn{2}{|c|}{ F6: habilidades de relación interpersonal } & 0,658 & & \\
\hline P8 & A mí, me resulta especialmente difícil dar apoyo emocional & & 0,616 & 0,853 \\
\hline P9 & $\begin{array}{l}\text { Tengo dificultades para establecer relaciones } \\
\text { interpersonales profundas y satisfactorias con algunas } \\
\text { personas }\end{array}$ & & 0,637 & 0,852 \\
\hline P11 & $\begin{array}{l}\text { Creo que tengo mucha capacidad para ponerme en el } \\
\text { lugar de los demás y comprender sus respuestas }\end{array}$ & & 0,626 & 0,852 \\
\hline P18 & Me considero «un/a buen/a psicólogo/a» & & 0,597 & 0,851 \\
\hline P20 & Creo que soy una persona sociable & & 0,633 & 0,852 \\
\hline P24 & $\begin{array}{l}\text { A mí, me resulta especialmente difícil entender los } \\
\text { sentimientos de los demás }\end{array}$ & & 0,631 & 0,853 \\
\hline P30 & $\begin{array}{l}\text { Tengo dificultades para relacionarme abiertamente con } \\
\text { mis profesores/jefes }\end{array}$ & & 0,615 & 0,850 \\
\hline \multicolumn{2}{|c|}{ Total del Cuestionario de Salud Mental Positiva } & 0,885 & & \\
\hline
\end{tabular}


Se analizó el índice de homogeneidad de todos los ítems del CSMP, tanto para el total como para cada factor. Se obtuvieron valores para el total del cuestionario superiores a 0,20 en todos los ítems, excepto en los siguientes: el 31 ("creo que soy un/a inútil y no sirvo para nada»), el 37 («me gusta ayudar a los demás»), el 5 («soy capaz de controlarme cuando experimento emociones negativas»), el 28 («delante de un problema, soy capaz de solicitar información») y el 35 («soy capaz de decir no cuando quiero decir no») (tabla 4).

\section{Validez convergente y discriminante}

En la tabla 5, se muestra el análisis de las correlaciones entre los factores y la puntuación total del cuestionario. Todas las correlaciones fueron significativas entre los factores y la puntuación total del CSMP. El factor 1 (satisfacción personal) y el factor 2 (actitud prosocial) se correlacionaron más fuertemente con la puntuación total $(r=0,666$ y $r=0,792$, respectivamente), mientras que el factor 5 (resolución de problemas y actualización) se correlaciona

Tabla 4. Correlación de cada ítem con el total del Cuestionario de Salud Mental Positiva

\begin{tabular}{|c|c|c|}
\hline Contenido de los ítems resumidos & $\begin{array}{c}\text { Correlación de } \\
\text { cada ítem con el } \\
\text { total de la escala } \\
\text { corregida }\end{array}$ & $\begin{array}{c}\text { Correlación de } \\
\text { cada ítem con el } \\
\text { total de la } \\
\text { subescala } \\
\text { corregida }\end{array}$ \\
\hline
\end{tabular}

\section{F1: satisfacción personal}

\begin{tabular}{|c|c|c|c|}
\hline P4 & Me gusto como soy & 0,234 & 0,235 \\
\hline P6 & Me siento a punto de explotar & 0,219 & 0,298 \\
\hline P7 & Para mí, la vida es aburrida y monótona & 0,390 & 0,379 \\
\hline P12 & Veo mi futuro con pesimismo & 0,447 & 0,492 \\
\hline P14 & $\begin{array}{l}\text { Me considero una persona menos importante que el resto } \\
\text { de personas que me rodean }\end{array}$ & 0,347 & 0,331 \\
\hline P31 & Creo que soy un/a inútil y no sirvo para nada & 0,165 & 0,223 \\
\hline P38 & Me siento insatisfecha/o conmigo misma/o & 0,241 & 0,303 \\
\hline P39 & Me siento insatisfecha/o de mi aspecto físico & 0,340 & 0,370 \\
\hline \multicolumn{4}{|c|}{ F2: actitud prosocial } \\
\hline P1 & $\begin{array}{l}\text { Me resulta especialmente difícil aceptar a los otros cuando } \\
\text { tienen actitudes distintas a las mías }\end{array}$ & 0,464 & 0,226 \\
\hline P3 & $\begin{array}{l}\text { A mí, me resulta especialmente difícil escuchar a las } \\
\text { personas que me cuentan sus problemas }\end{array}$ & 0,512 & 0,219 \\
\hline P23 & Pienso que soy una persona digna de confianza & 0,319 & 0,163 \\
\hline P25 & Pienso en las necesidades de los demás & 0,263 & 0,152 \\
\hline P37 & Me gusta ayudar a los demás & 0,150 & 0,054 \\
\hline
\end{tabular}

Continúa 
Tabla 4. Correlación de cada ítem con el total del Cuestionario de Salud Mental Positiva (cont.)

Contenido de los ítems resumidos

\begin{tabular}{c|c}
$\begin{array}{c}\text { Correlación de } \\
\text { cada ítem con el } \\
\text { total de la escala } \\
\text { corregida }\end{array}$ & $\begin{array}{c}\text { Correlación de } \\
\text { cada ítem con el } \\
\text { total de la } \\
\text { subescala } \\
\text { corregida }\end{array}$ \\
\hline
\end{tabular}

F3: autocontrol

\begin{tabular}{|l|l|l|l|}
\hline P2 & Los problemas me bloquean fácilmente & 0,407 & $-0,039$ \\
\hline P5 & $\begin{array}{l}\text { Soy capaz de controlarme cuando experimento emociones } \\
\text { negativas }\end{array}$ & 0,165 & 0,318 \\
\hline P21 & $\begin{array}{l}\text { Soy capaz de controlarme cuando tengo pensamientos } \\
\text { negativos }\end{array}$ & 0,375 & 0,605 \\
\hline P22 & $\begin{array}{l}\text { Soy capaz de mantener un buen nivel de autocontrol en las } \\
\text { situaciones conflictivas de mi vida }\end{array}$ & 0,486 & 0,679 \\
\hline P26 & $\begin{array}{l}\text { Si estoy viviendo presiones exteriores desfavorables, soy } \\
\text { capaz de continuar manteniendo mi equilibrio personal }\end{array}$ & 0,285 & 0,588 \\
\hline
\end{tabular}

F4: autonomía

\begin{tabular}{|l|l|l|l|}
\hline P10 & Me preocupa mucho lo que los demás piensan de mí & 0,415 & 0,624 \\
\hline P13 & $\begin{array}{l}\text { Las opiniones de los demás me influyen mucho a la hora } \\
\text { de tomar mis decisiones }\end{array}$ & 0,542 & 0,702 \\
\hline P19 & Me preocupa que la gente me critique & 0,279 & 0,539 \\
\hline P33 & Me resulta difícil tener opiniones personales & 0,484 & 0,632 \\
\hline P34 & $\begin{array}{l}\text { Cuando tengo que tomar decisiones importantes, me siento } \\
\text { muy insegura/o }\end{array}$ & 0,398 & 0,673 \\
\hline
\end{tabular}

F5: resolución de problemas y actualización

\begin{tabular}{|l|l|l|l|}
\hline P15 & Soy capaz de tomar decisiones por mí misma/o & 0,383 & 0,660 \\
\hline P16 & $\begin{array}{l}\text { Intento sacar los aspectos positivos de las cosas malas que } \\
\text { me suceden }\end{array}$ & 0,363 & 0,546 \\
\hline P17 & Intento mejorar como persona & 0,403 & 0,515 \\
\hline P27 & Cuando hay cambios en mi entorno, intento adaptarme & 0,405 & 0,609 \\
\hline P28 & Delante de un problema, soy capaz de solicitar información & 0,131 & 0,299 \\
\hline P29 & Los cambios que ocurren en mi rutina habitual me estimulan & 0,231 & 0,305 \\
\hline P32 & Trato de desarrollar y potenciar mis buenas aptitudes & 0,402 & 0,494 \\
\hline P35 & Soy capaz de decir no cuando quiero decir no & 0,122 & 0,088 \\
\hline P36 & $\begin{array}{l}\text { Cuando se me plantea un problema, intento buscar posibles } \\
\text { soluciones }\end{array}$ & 0,457 & 0,664 \\
\hline
\end{tabular}


Tabla 4. Correlación de cada ítem con el total del Cuestionario de Salud Mental Positiva (cont.)

\begin{tabular}{|c|c|c|}
\hline Contenido de los ítems resumidos & $\begin{array}{c}\text { Correlación de } \\
\text { cada ítem con el } \\
\text { total de la escala } \\
\text { corregida }\end{array}$ & $\begin{array}{c}\text { Correlación de } \\
\text { cada ítem con el } \\
\text { total de la } \\
\text { subescala } \\
\text { corregida }\end{array}$ \\
\hline
\end{tabular}

F6: habilidades de relación interpersonal

\begin{tabular}{|l|l|l|l|}
\hline P8 & A mí, me resulta especialmente difícil dar apoyo emocional & 0,281 & 0,389 \\
\hline P9 & $\begin{array}{l}\text { Tengo dificultades para establecer relaciones } \\
\text { interpersonales profundas y satisfactorias con algunas } \\
\text { personas }\end{array}$ & 0,331 & 0,319 \\
\hline P11 & $\begin{array}{l}\text { Creo que tengo mucha capacidad para ponerme en el lugar } \\
\text { de los demás y comprender sus respuestas }\end{array}$ & 0,309 & 0,356 \\
\hline P18 & Me considero «un/a buen/a psicólogo/a» & 0,364 & 0,444 \\
\hline P20 & Creo que soy una persona sociable & 0,327 & 0,332 \\
\hline P24 & $\begin{array}{l}\text { A mí, me resulta especialmente difícil entender los } \\
\text { sentimientos de los demás }\end{array}$ & 0,340 \\
\hline P30 & $\begin{array}{l}\text { Tengo dificultades para relacionarme abiertamente con mis } \\
\text { profesores/jefes }\end{array}$ & 0,390 & 0,393 \\
\hline
\end{tabular}

Tabla 5. Correlaciones del Cuestionario de Salud Mental Positiva (subescalas y escala total)

\begin{tabular}{|l|c|c|c|c|c|c|}
\hline \multicolumn{7}{|c|}{ Tabla 5. Correlaciones del Cuestionario de Salud Mental Positiva (subescalas y escala total) } \\
\hline
\end{tabular}

*Todas las correlaciones son significativas; nivel de significación $p<0,01$. 
más débilmente con la puntuación total $(r=0,617)$. La correlación más fuerte entre los factores se observó entre el factor 5 (resolución de problemas y actualización) y el factor 3 (autocontrol) $(r=0,593)$, mientras que la correlación más débil fue entre el factor 4 (autonomía) y el factor 5 (resolución de problemas y actualización) ( $r=0,007)$.

En el análisis de la validez discriminante, se encontraron diferencias estadísticamente significativas para la puntuación total del cuestionario y en cuatro de las seis dimensiones del CSMP, en el sentido de que las puntuaciones medias fueron más altas en el grupo con GHQ-12 superior a 2 (tabla 6).

\section{DISCUSIÓN}

Este estudio ha permitido evaluar la fiabilidad y validez del CSMP sobre una muestra de profesores universitarios de Enfermería.

Los resultados revelan que las características psicométricas del CSMP son favorables.

En relación con la fiabilidad, los resultados obtenidos mostraron que cinco de los seis factores propuestos (F1, F3, F4, F5 y F6) obtuvieron un buen nivel de fiabilidad en términos de consistencia interna, teniendo en cuenta el trabajo publicado por Fortin et al. ${ }^{16}$, en el que se recomiendan valores superiores a 0,60 para considerar un nivel aceptable de fiabilidad de la medida. El factor F2 (actitud prosocial) es el factor que ha obtenido un coeficiente alfa de Cronbach más bajo tanto en este estudio como en otras investigaciones, donde los resultados han oscilado entre valores límite de 0,60 en el estudio de Lluch et al. (2013), realizado en una muestra de personas con trastornos crónicos de salud física, y de 0,51 obtenido por Sequeira et al. (2014) en una muestra de estudiantes universitarios de Enfermería portugueses.

Todo esto permite pensar que el CSMP tiene un buen nivel de fiabilidad para la medición del constructo de SMP.

En la muestra estudiada, los resultados obtenidos en el análisis de los ítems del CSMP fueron más elevados (intervalo de 1,90 del ítem 34 a 3,67 del ítem 23) que los obtenidos en el estudio de Albacar ${ }^{17}$ (2014) (intervalo de 1,22 del ítem 23 a 2,31 del ítem 18). Además, el intervalo de la DE de este estudio fue ligeramente inferior al obtenido en el estudio de

Tabla 6. Validez discriminante del Cuestionario de Salud Mental Positiva (CSMP)

\begin{tabular}{|l|c|c|c|}
\hline & $\begin{array}{c}\text { Goldberg } \leq 2 \\
\text { Media (DE) }\end{array}$ & $\begin{array}{c}\text { Goldberg }>2 \\
\text { Media (DE) }\end{array}$ & \multirow{2}{*}{$p^{*}$} \\
\hline F1: satisfacción personal & $\mathrm{n}=192$ & $\mathrm{n}=71$ & \\
\hline F2: actitud prosocial & $23,4(3,2)$ & $22,2(2,7)$ & 0,005 \\
\hline F3: autocontrol & $14,8(2,3)$ & $14,1(1,6)$ & 0,011 \\
\hline F4: autonomía & $15,4(2,5)$ & $14,9(2,2)$ & 0,120 \\
\hline F5: resolución de problemas y autoactualización & $10,3(3,8)$ & $9,7(3,3)$ & 0,249 \\
\hline F6: habilidades de relación interpersonal & $30,4(3,8)$ & $28,8(3,3)$ & 0,003 \\
\hline Total CSMP & $21,9(3,1)$ & $20,2(2,3)$ & 0,0001 \\
\hline & $116,5(12,5)$ & $110,3(9,2)$ & 0,0001 \\
\hline
\end{tabular}

${ }^{*} t$ de Student-Fisher; DE: desviación estándar; n: tamaño de la muestra; p: nivel de significación. 
Albacar. En el presente estudio, osciló entre 0,59 (ítem 37) y 1,06 (ítem 3 e ítem 19) y, en el estudio de Albacar, osciló entre 0,53 (ítem 23) y 1,13 (ítem 35).

La validez discriminante mostró que el CSMP fue capaz de discriminar entre docentes con diferentes niveles del GHQ-12.

En este estudio, una posible limitación es que la muestra utilizada es una población procedente de una zona determinada de España (Comunidad Autónoma de Cataluña), y puede no ser representativa de otras comunidades de España.

\section{CONCLUSIONES}

Los resultados de este estudio ponen de manifiesto como el CSMP ha mostrado ser un instrumento adecuado para valorar el nivel de SMP en docentes universitarios de Enfermería. Ha demostrado tener adecuadas propiedades métricas tanto para el constructo global de la escala como para los seis factores que lo configuran. La consistencia interna ha sido favorable para cinco de los seis factores que configuran la estructura del cuestionario: F1 (satisfacción personal), F3 (autocontrol), F4 (autonomía), F5 (resolución de problemas y autoactualización) y F6 (habilidades de relación interpersonal). El factor F2 (actitud prosocial) ha mostrado valores de fiabilidad más moderados, que deberán ser comprobados en posteriores estudios.

\section{BIBLIOGRAFÍA}

1. World Health Organization (WHO). Strengthening mental health promotion. Fact Sheet No. 220. Ginebra: WHO; 2001.

2. Bolier L, Haverman M, Westerhof GJ, Riper H, Smit F, Bohlmeijer E. Positive psychology interventions: a meta-analysis of randomized controlled studies. BMC Public Health. 2013;13:119.

3. Aspinwall LG, Tedeschi RG. The value of positive psychology for health psychology: progress and pitfalls in examining the relation of positive phenomena to health. Ann Behav Med. 2010;39(1):4-15.
4. Lluch MT. Construcción de una escala para evaluar la salud mental positiva. Tesis doctoral. Barcelona: Facultad de Psicología, Universitat de Barcelona; 1999.

5. Jahoda M. Current concepts of positive mental health. Nueva York: Basic Books; 1958.

6. Lluch MT. Construcción y análisis psicométrico de un cuestionario para evaluar la salud mental positiva. Psic Conductual. 2003;11(1):61-78.

7. Amar J, Palacio J, Llinás H, Puerta L, Sierra E, Pérez AM, et al. Calidad de vida y salud mental positiva en menores trabajadores de Toluviejo. Suma Psicol. 2008; 15(2):385-403.

8. Lluch-Canut T, Puig-Llobet M, Sánchez-Ortega A, Roldán-Merino J, Ferré-Grau C; Positive Mental Health Research Group (PMHRG). Assessing positive mental health in people with chronic physical health problems: correlations with socio-demographic variables and physical health status. BMC Public Health. 2013; 13:928.

9. Navarro GL. Características de salud mental positiva en estudiantes del programa de Medicina de la Universidad Libre Seccional Barranquilla. Academia Libre. 2009; (7):93-102.

10. Ospino CM. Caracterización de la salud mental positiva de menores trabajadores en un mercado público. Trabajo de fin de máster. Barranquilla: Universidad del Norte; 2012.

11. Sequeira C, Carvalho JC, Sampaio F, Sá L, Lluch-Canut T, Roldán-Merino J. Avaliação das propriedades psicométricas do Questionário de Saúde Mental Positiva em estudantes portugueses do ensino superior. Rev Port Enferm Saúde Ment. 2014;(11):45-53.

12. Meltzer $\mathrm{H}$. Development of a common instrument for mental health. En: Nosikov A, Gudex C (eds). EUROHIS: Developing Common Instruments for Health Surveys. Ámsterdam: IOS Press; 2003. p. 35-60.

13. Sánchez-López MP, Dresch V. The 12-Item General Health Questionnaire (GHQ-12): reliability, external validity and factor structure in the Spanish population. Psicothema. 2008;20(4):839-43.

14. Clark LA, Watson D. Constructing validity: basic issues in objective scale development. Psychol Assess. 1995; 7(3):309-19.

15. Fayers PM, Machin D. Quality of life: assessment, analysis and interpretation. Londres: John Wiley \& Sons; 2000.

16. Fortin MF, Côté J, Filion F. Fundamentos e etapas do processo de investigação. Loures: Lusodidacta; 2009.

17. Albacar N. Atenció d'infermeria a la cuidadora principal de persones amb esquizofrènia: valoració dels requisits d'autocura i de la salut mental positiva. [Tesis]. Tarragona: Universitat Rovira i Virgili; 2014. 